\title{
Monopole ordered phases in dipolar and nearest-neighbors Ising pyrochlore: From spin ice to the all-in-all-out antiferromagnet
}

\author{
P. C. Guruciaga, ${ }^{1, *}$ S. A. Grigera, ${ }^{2,3}$ and R. A. Borzi ${ }^{4}$ \\ ${ }^{1}$ Instituto de Investigaciones Físicas de Mar del Plata (IFIMAR), UNMdP-CONICET and Departamento de Física, Facultad de Ciencias \\ Exactas y Naturales, Universidad Nacional de Mar del Plata, Dean Funes 3350, 7600 Mar del Plata, Argentina \\ ${ }^{2}$ Instituto de Física de Líquidos y Sistemas Biológicos (IFLYSIB), UNLP-CONICET, 1900 La Plata, Argentina \\ ${ }^{3}$ School of Physics and Astronomy, University of St Andrews, St Andrews KY16 9SS, United Kingdom \\ ${ }^{4}$ Instituto de Investigaciones Fisicoquímicas Teóricas y Aplicadas (INIFTA), UNLP-CONICET and Departamento de Física, \\ Facultad de Ciencias Exactas, Universidad Nacional de La Plata, c.c. 16, suc. 4, 1900 La Plata, Argentina \\ (Received 7 September 2014; revised manuscript received 30 October 2014; published 21 November 2014)
}

\begin{abstract}
We study Ising pyrochlores by means of Monte Carlo simulations. We cover a set of exchange constants ranging from the frustrated ferromagnetic case (spin-ice) to the fully-ordered "all-in-all-out" antiferromagnet in the dipolar model, reinterpreting the results—as in an ionic system-in terms of a temperature vs magnetic charge density phase diagram. In spite of its spin nature and the presence of both double and single nonconserved magnetic charges, the dipolar model gives place to a phase diagram which is quite comparable with those previously obtained for on-lattice systems of electric charges, and on spin ice models with a conserved number of single magnetic charges. The contrast between these systems, to which we add results from the nearest-neighbors model, put forward other features of our phase diagram—notably, a monopole fluid with charge order at high monopole densities that persists up to arbitrarily high temperatures - that can only be explained taking into account construction constraints forced by the underlying spin degrees of freedom.
\end{abstract}

DOI: 10.1103/PhysRevB.90.184423

PACS number(s): 75.10.Hk, 02.70.Uu, 75.50.-y

\section{INTRODUCTION}

Describing a complex material in terms of low-lying particlelike collective excitations-quasiparticles-is one of the key approaches in condensed matter physics [1]. Phonons, magnons, electrons, and holes in semiconductors are some of the better known examples of these excitations. But quasiparticles form a very rich set, including topological excitations and fractionalization. In a first approximation, these excitations are considered as noninteracting, with all the complexity of the system hidden in the quasiparticles themselves. The next layer of description, where interactions between quasiparticles are included, leads to a great variety of behaviors: from anharmonic effects in crystals to the stabilization of higher hierarchies of order such as magnon binding [2] or magnonmediated heavy fermion superconductivity [3]. Recently, a new kind of fractional pointlike topological excitation has been proposed theoretically [4], and evidence of its existence was found experimentally [5-7] in the spin ice compounds. These new quasiparticles are sources of magnetic field and interact via a Coulomb-like potential, hence their name magnetic monopoles [4]. This approach allows for a very effective description of the thermodynamics, the dynamics, and the out of equilibrium behavior of spin-ice systems $[4,8-15]$.

The magnetic properties of spin ice materials can be described by classical magnetic moments in a pyrochlore lattice, occupying the vertices of corner-sharing tetrahedra. They behave at low temperatures as Ising-like spins [16] $\boldsymbol{\mu}_{i}=\mu S_{i} \hat{\mathbf{e}}_{i}$ with $S_{i}= \pm 1$, pointing along the $\langle 111\rangle$ directions $\hat{\mathbf{e}}_{i}$ (Fig. 1). The magnetic interactions of exchange and dipolar origin, of strengths $J$ and $D$, respectively, are well accounted

\footnotetext{
*Corresponding author: pguruciaga@ifimar-conicet.gob.ar
}

for by the dipolar spin ice model (DSIM) Hamiltonian:

$$
\begin{aligned}
\frac{\mathcal{H}}{T}= & \frac{D}{T}\left(\frac{J}{3 D} \sum_{\langle i j\rangle} S_{i} S_{j}\right. \\
& \left.+a^{3} \sum_{(i, j)}\left[\frac{\hat{\mathbf{e}}_{i} \cdot \hat{\mathbf{e}}_{j}}{\left|\mathbf{r}_{i j}\right|^{3}}-\frac{3\left(\hat{\mathbf{e}}_{i} \cdot \mathbf{r}_{i j}\right)\left(\hat{\mathbf{e}}_{j} \cdot \mathbf{r}_{i j}\right)}{\left|\mathbf{r}_{i j}\right|^{5}}\right] S_{i} S_{j}\right),
\end{aligned}
$$

where $T$ is the temperature, $a$ is the lattice spacing, $\langle i j\rangle$ means that the sum is carried over nearest neighbors, $\left|\mathbf{r}_{i j}\right|$ is the distance between spins $i$ and $j$, and $D=\mu_{0} \mu^{2} /\left(4 \pi a^{3}\right)$.

The nearest neighbors spin ice model (NNSIM) Hamiltonian is derived from the DSIM by keeping only the nearest neighbor contributions of the dipolar interaction:

$$
\frac{\mathcal{H}_{n n}}{T}=\frac{J_{n n}+D_{n n}}{T} \sum_{\langle i j\rangle} S_{i} S_{j}
$$

with $J_{n n}=J / 3$ and $D_{n n}=5 D / 3$, i.e., an effective exchange interaction of strength $J_{\text {eff }}=J_{n n}+D_{n n}$. For the spin ice materials, $J_{\text {eff }}$ in (2) is positive $\left(J_{n n} / D_{n n}>-1\right)$ and imposes the spin-ice rule (named after Bernal and Fowler's ice rules [17]): Two spins should point in and two out of a tetrahedron. This rule can be translated into field theory language as a divergence free condition, which gives rise to a "Coulomb phase" [18]. Following Ref. [4], a violation of this law can be interpreted as the creation of a charge-a monopole - sitting in the tetrahedron; within the DSIM, a Coulomb-like magnetic charge proportional to the divergence of the spin vectors can be associated with each of these excitations. Single excitations of opposite signs are related to "3-in/1-out" or "1-in/3-out" configurations, while double excitations (with double charge) correspond to the "all-in" or "all-out" configurations. The antiferromagnetic version 


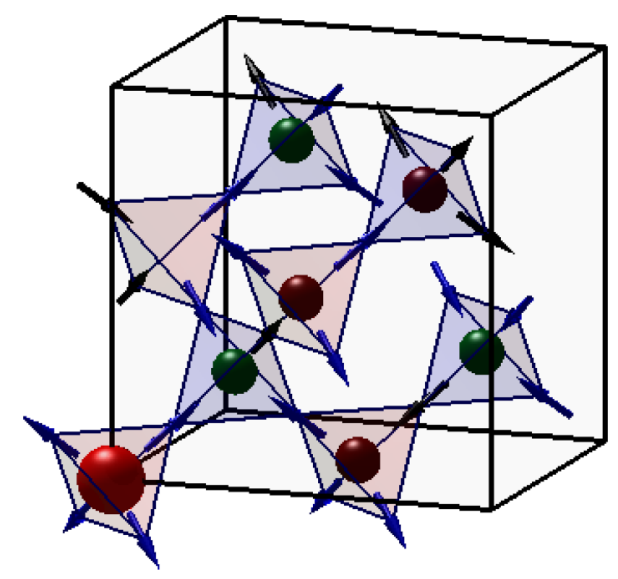

FIG. 1. (Color online) Conventional unit cell of the pyrochlore lattice. In spin ice compounds, Ising-like spins occupy the vertices of corner-sharing "up" (pink) and "down" (lilac) tetrahedra. Black/blue spins point inwards/outwards (along the local $\langle 111\rangle$ directions) of an up tetrahedron. Via the dumbbell model [4], the diverse spin configurations are mapped to different types of magnetic charges: single/double (small/big) and positive/negative (green/red) monopoles (note that since this unit cell is only a part of a bigger system, the constraint of local magnetic neutrality is not satisfied). This configuration snapshot was obtained for a material with $J_{n n} / D_{n n}=-0.947$ at $T / D_{n n} \approx 0.57$, resulting in a monopole density (defined as the average number of charges per tetrahedron) of $\rho \approx 1.1$.

of this model, with negative $J_{\text {eff }}$ (i.e., $J_{n n} / D_{n n}<-1$ ), has an unfrustrated ground state corresponding precisely to an ordered zinc-blende structure of double charges: Spins in alternating tetrahedra are in configurations "all-in" and "allout." Though much searched for, no Ising pyrochlore with this spin ordering has been found yet [19].

At zero magnetic field the density of monopoles is regulated by the sign and magnitude of $J_{\text {eff }} / T$ which, in the currently known materials, leads at best to moderately correlated monopole fluids [20,21]. In order to explicitly show the effect of these correlations at low temperatures while stressing the role of charge degrees of freedom, some of us recently reported the results of simulations on a dipolar spin ice model where a new ingredient was introduced: While keeping the dipolar Hamiltonian (1), our approach in the conserved monopole dipolar spin ice model (CDSIM) was to use the density of conserved single monopoles as the main control parameter [22]. Our finding of phases with different degrees of long range chargelike ordering reinforced the beauty and simplicity of the monopolar scenario introduced by Castelnovo and collaborators [4]. A very recent contribution addresses this same issue in a wider scope [23]. Excluding double charges in the dumbbell model (which-unlike the previous approachtakes magnetic charges and not spins as their interacting simple entities), they show that a Coulomb phase can still be defined beneath a crystal of magnetic single charges. One drawback of these approaches is that the explicit omission of double defects is somewhat contrived: In real materials, the limit $J_{\text {eff }} / T \rightarrow 0$ implies the proliferation of both single and double excitations. Furthermore, within the CDSIM model, the ordered ground state expected for negative $J_{\text {eff }} / T$ is not allowed [24].
In this paper we return to the usual DSIM in order to address these shortcomings in the previous analysis and to extend it in order to include the antiferromagnetic case. Building up on previous results by den Hertog et al. [24], we show that when examined using the framework of monopolar excitations both the DSIM and the CDSIM lead to the same physics, and in particular to very similar phase diagrams. Since within the NNSIM one would naively expect no monopole-monopole interaction, one would think that charge degrees of freedom play no role. In spite of this, we will see that the phase diagram obtained for the NNSIM model can be reinterpreted in terms of effective nearest-neighbors interactions between double charges. We will also show that these effective interactions (neither dipolar nor exchange in origin, but arising from correlations imposed by the internal degrees of freedom of the charges) also affect the phase diagram in the presence of dipolar interactions.

\section{Simulation details}

We performed Monte Carlo simulations with a single spin-flip Metropolis algorithm, using Ewald summations to take into account the long-range interactions [25]. We used a conventional cubic cell for the pyrochlore lattice, which contains 16 spins, and simulated systems with $L \times L \times L$ cells. Thermodynamic data were collected by starting at high temperatures and cooling very slowly, for different values of $J_{n n} / D_{n n}$ (as this ratio will be negative throughout this paper, we will usually refer to its absolute value). Typically, we needed $10^{4}$ Monte Carlo steps for equilibration and $2 \times 10^{4}$ for averaging at each temperature for $L=4$, but we used up to $10^{5}$ steps for bigger lattices. First order transition points deserve to be mentioned separately; there, full equilibration was only achieved after longer times (up to $5 \times 10^{5}$ Monte Carlo steps) for sizes below $L=5$.

\section{CHARGE DEGREES OF FREEDOM IN THE DIPOLAR MODEL}

Figure 2 shows the monopole density $\rho$ as a function of temperature for $L=4$. We define $\rho$ as the number of single charges per unit tetrahedron, thus counting double monopoles as the superposition of two single charges of the same sign in a tetrahedron. In a similar way to Fig. 2 of Ref. [23], where only single charges were considered, we can distinguish two families of curves according to their limit as $T \rightarrow 0$. The first type, which tend to $\rho=0$ (with $\left|J_{n n} / D_{n n}\right| \leqslant 0.911$ ), were characterized as spin ices by determining their residual entropy, via numerical integration of the specific heat divided by temperature. The second type are curves that tend to $\rho=2$ when $T \rightarrow 0$, meaning that they reach a state in which all the tetrahedra are occupied by double monopoles (i.e., the nonfrustrated antiferromagnetic phase). For certain values of $\left|J_{n n} / D_{n n}\right|$ in the latter group of curves, the monopole density per tetrahedron suffers a sudden change at low temperatures from $\rho \approx 0$ to $\rho \approx 2$. It is straightforward to associate the appearance of this zinc-blende structure [22] with the presence of Coulomb-like interactions between monopoles. However, later we will show that this phase (which is no other than the "all-in-all-out" phase mentioned in the title and in the 


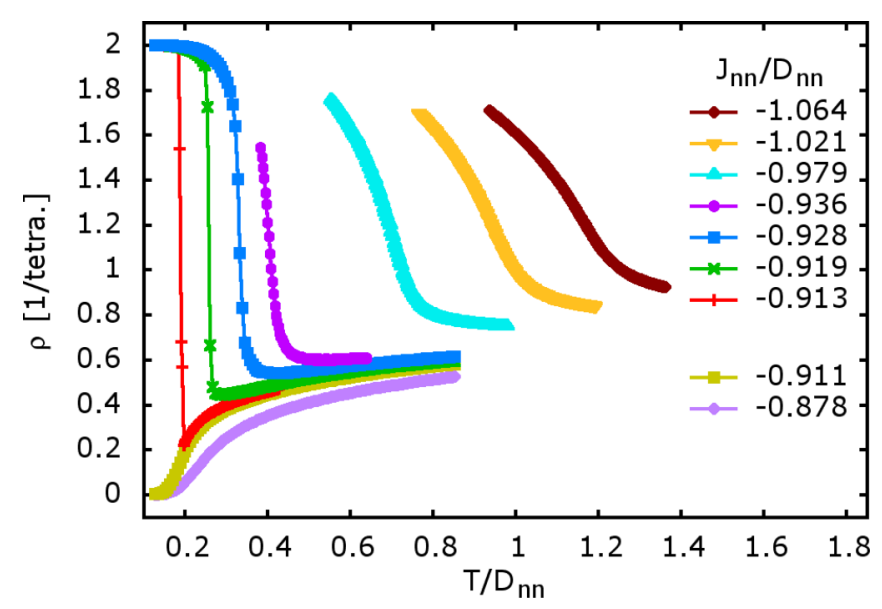

FIG. 2. (Color online) Number of single charges per tetrahedron vs temperature for $L=4$ and different values of $J_{n n} / D_{n n}$. It is possible to distinguish two very different regimes: Some of the curves (those with $\left|J_{n n} / D_{n n}\right| \leqslant 0.911$ ) reach $\rho=0$ when $T \rightarrow 0$, while the rest approach $\rho=2$. Among the latter it is easy to notice that the jump between low and high density becomes smoother as $\left|J_{n n} / D_{n n}\right|$ increases.

introduction) can also be stabilized in the NNSIM, where these interactions are absent.

The curves in Fig. 3 (upper panel) represent the molar specific heat $C$ as a function of temperature for different values of $\left|J_{n n} / D_{n n}\right| \geqslant 0.913$ and $L=4$ (i.e., corresponding only to curves in the second branch of Fig. 2). We see sharp, deltalike peaks that become wider and shorter as $\left|J_{n n} / D_{n n}\right|$

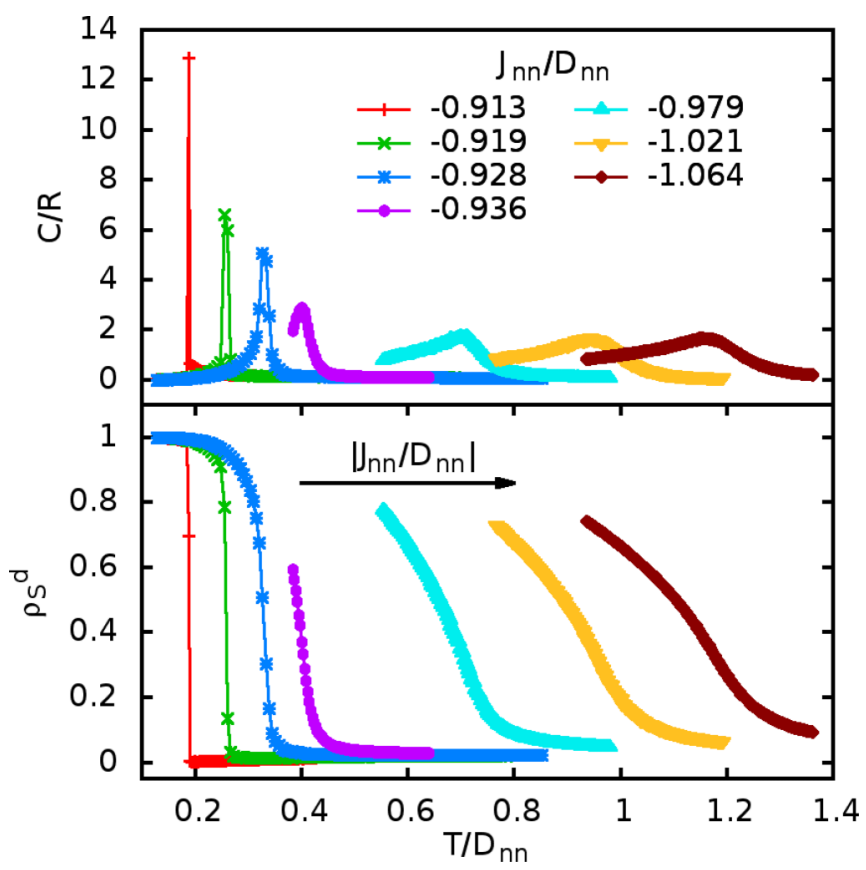

FIG. 3. (Color online) Top: molar specific heat for $L=4$ as a function of temperature; the sharp, deltalike peak broadens as $\left|J_{n n} / D_{n n}\right|$ increases-a sign of a first order phase transition that becomes second order. This idea is reinforced by the double monopole staggered density (bottom), which displays a steplike jump that becomes smooth and continuous. increases, correlated with the jumps we noticed in $\rho$. With the development of a zinc-blende structure in mind, we also present our results of the double monopole staggered density $\rho_{S}^{d}$, defined as the average of the modulus of the total magnetic charge due to double monopoles in up tetrahedra per sublattice site per unit charge. A nearly zero value of this quantity implies that there is no symmetry breaking between the up and down tetrahedra sublattices, while $\rho_{S}^{d} \approx 1$ is the result of a staggered ordering of the magnetic charges: positive and negative double monopoles alternating in the sublattices, occupying all the tetrahedra. In Fig. 3 (lower panel) we observe a jump between these two states, which is sudden and steplike for low values of $\left|J_{n n} / D_{n n}\right|$ and slowly changes into continuous as that ratio increases. It is interesting to note that these steplike jumps are correlated to the ones in $\rho$ : The system becomes dense and charge ordered suddenly and simultaneously, just as it happens in the crystallization transition of ionic fluids. On the other hand, the continuous developing of both the staggered density and the number density at higher $\left|J_{n n} / D_{n n}\right|$ indicates the transition to a phase in which the local density is homogeneous (fluidlike) but charge ordered.

To confirm the existence of these transitions and determine their order we performed finite size analysis over values of $J_{n n} / D_{n n}$ representative of the two behaviors. In Fig. 4 we present our results of the molar specific heat for $J_{n n} / D_{n n}=$ -0.919 . The previous suggestion of a first order transition taking place for these values of $J_{n n} / D_{n n}$ is backed up by the fact that the value of the specific heat and the double monopole susceptibility $\chi_{S}^{d}$ (defined as the fluctuations of the corresponding staggered density over the temperature) at their maximum are proportional to the volume of the system (Fig. 4 , inset). We also studied $J_{n n} / D_{n n}=-1.064$, in which the double monopole susceptibility (Fig. 5), as well as the specific heat and the double monopole staggered density (not shown), evolve with the size of the system as in a second order phase transition. The critical exponents are consistent with the three-dimensional Ising universality class (Fig. 5, inset).

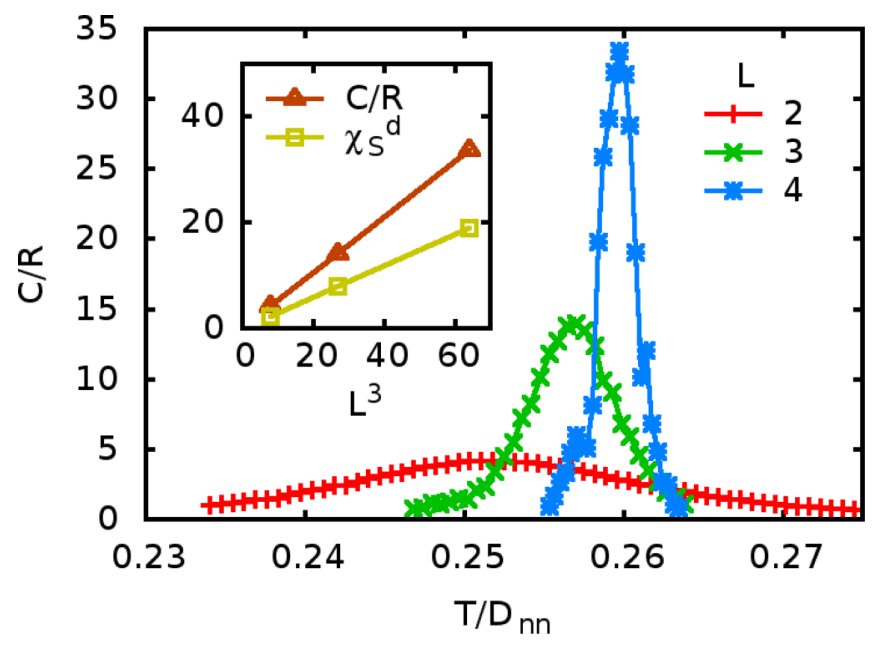

FIG. 4. (Color online) For $J_{n n} / D_{n n}=-0.919$ the specific heat data displays finite size effects consistent with a first order phase transition. Moreover, the value of the specific heat and the double monopole susceptibility at their maximum grow linearly with the volume of the system, as shown in the inset. 


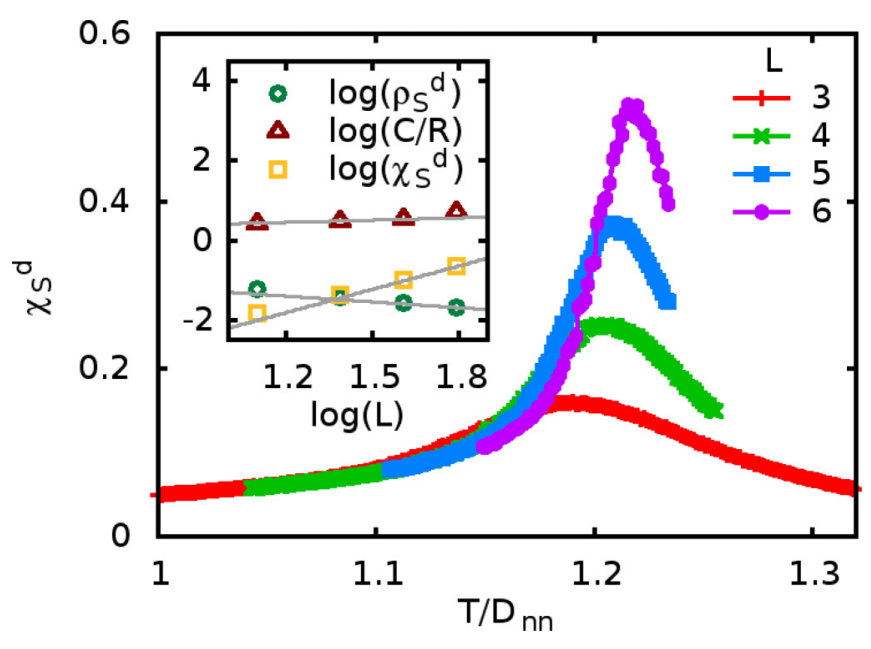

FIG. 5. (Color online) For $J_{n n} / D_{n n}=-1.064$ the double monopole susceptibility displays finite size effects consistent with a second order phase transition. The values of $\rho_{S}^{d}\left(T_{c}(L)\right), C\left(T_{c}(L)\right) / R$, and $\chi_{S}^{d}\left(T_{c}(L)\right)$ evolve as power laws with the size of the system and present good correspondence with the behavior expected for the three dimensional Ising model universality class [27] (gray lines).

Since this line of second order transitions becomes first order, there must be a tricritical point of the Blume-Emery-Griffiths universality class [26].

A nice way to summarize all the previous results while emphasizing the importance of charge degrees of freedom is to construct a phase diagram in terms of the monopole density and the temperature (Fig. 6). The color map corresponds to the interpolated value of the double monopole staggered density $\rho_{S}^{d}$ as a function of temperature and monopole number density (for $L=4$ ). The graph was obtained by combining a set

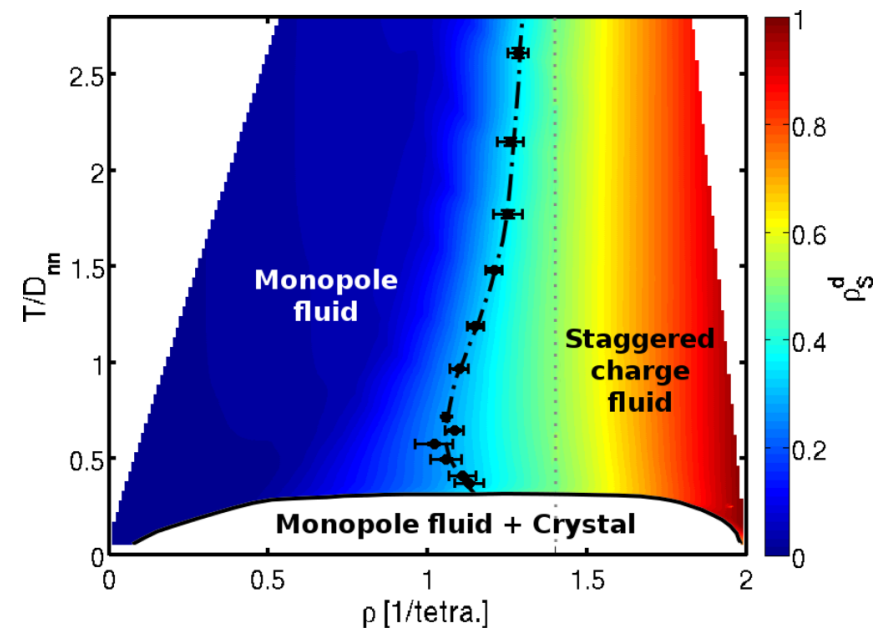

FIG. 6. (Color online) Temperature vs monopole density phase diagram with dipolar (long-range) interactions. At low temperatures the system undergoes a first order phase transition (white dome), where a low density gas and a crystal coexist. The transition becomes second order (dot-dashed line) at a tricritical point near $\rho_{t} \approx 1.17$, $T_{t} / D_{n n} \approx 0.34$. The diagram is overlaid on top of an interpolated contour plot of $\rho_{S}^{d}$ for $L=4$. The vertical dotted line at $\rho_{c} \approx 1.4$ indicates the critical density of the geometric transition in the NNSIM (see Sec. IV A). of more than thirty $\rho_{S}^{d}$ vs $T$ curves (of which those shown in Fig. 3, bottom, constitute a subset) with $\rho$ vs $T$ curves (like those in Fig. 2). The filled circles represent the location of the maximum on the specific heat for various values of $\left|J_{n n} / D_{n n}\right|$ (see Fig. 3, top). Note that the vertical asymptote observed for this curve at high temperature corresponds to the limit in which antiferromagnetic exchange dominates over the dipolar interactions. The white dome (drawn by estimating the region where discontinuities would be observed for the infinite system) represents a forbidden region in parameter space in which, in the thermodynamic limit $L \rightarrow \infty$, the system cannot stay homogeneous. A system with $\rho=0.4$ at $T / D_{n n} \approx 0.22$ is exactly at the edge of the dome; if cooled down, it would separate into two phases occupying different parts of the material. On one hand, we would get a low-density monopole fluid, in which most of the tetrahedra are empty. On the other hand, a phase with a high double monopole density would crystallize into the zinc-blende ionic structure. In a tricritical point around $\rho_{t} \approx 1.17$ and $T_{t} / D_{n n} \approx 0.34$ the first order transition becomes second order, separating the monopole fluid and a staggered charge fluid in which local density is homogeneous but negative and positive charges display a tendency to occupy different sublattices, thus breaking the symmetry without phase separation [28]. It is interesting to note that both this staggered charge fluid and the crystal phase are more natural, double charge analogs of the single monopole ordered fluid and crystal found in the slightly artificial CDSIM (see Fig. 4 of Ref. [22]). The apparent differences between this phase diagram and that presented in Fig. 1 of Ref. [24] (of which we give our own version in Fig. 9) should not mask the fact that they both describe the same physics.

On the other hand, despite the great similarity between the phase diagram of Fig. 6 and that for the CDSIM, several differences arise. Since we used a single spin flip algorithm and did not fix the number of monopolar defects, the MelkoGingras-den Hertog first order transition [25] at $T / D_{n n}=$ 0.077 to an "ordered vacuum" of magnetic charges [22] is not visible. Also, Fig. 6 exhibits a re-entrant behavior of the monopole fluid at temperatures just above $T_{t} / D_{n n}$. Finally, one would expect that a disordered phase should always be found at high temperatures $\left(T / D_{n n} \gg 1\right)$ for any charge density, when entropic forces overcome monopole attraction. While this is true both for a system of real Coulomb charges in a lattice [29] and for the CDSIM [22], we do not see the line of second order transitions joining $\rho=2$ in the present case. Instead, this line reaches an asymptotically vertical behavior, parallel to the temperature axis. This fact, which seems to point to an infinitely high interaction energy between charges, could hardly be explained by the monopolar Hamiltonian on its own [4]. These last two cases deserve special attention, and will be analyzed in depth in the next sections.

\section{COMPARISON BETWEEN THE DIPOLAR AND THE NEAREST-NEIGHBORS MODEL}

In order to better understand the limits of the magnetic charges picture and the energetics of the models we have introduced, we also studied the NNSIM [Eq. (2)] for different ratios $J_{n n} / D_{n n}$. Similar to the previous case, we found a peak in the specific heat and the double monopole susceptibility, 


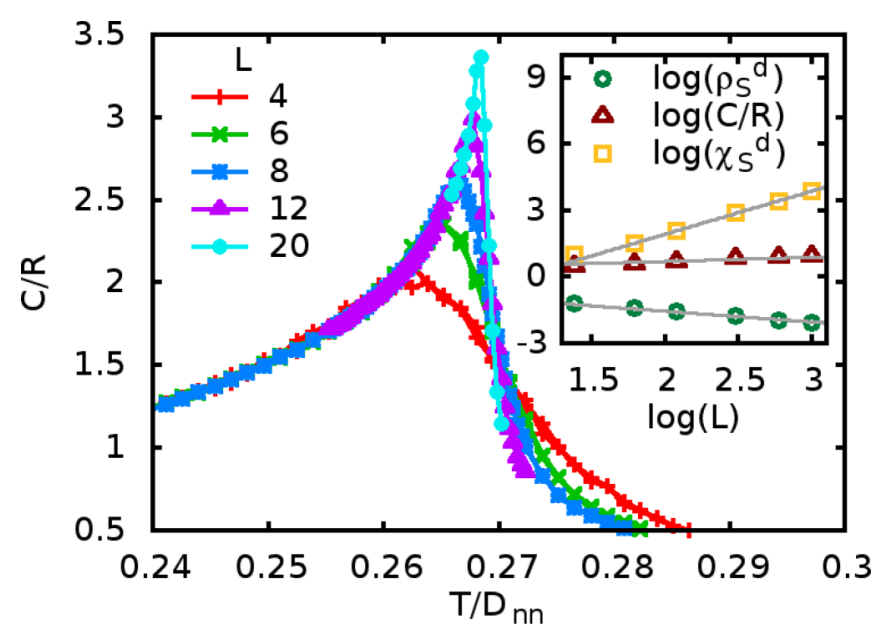

FIG. 7. (Color online) In the case of the nearest neighbor model, the specific heat displays the finite size effects characteristic of a second order phase transition for $J_{n n} / D_{n n}=-1.064$. Inset: again, the values of $\rho_{S}^{d}\left(T_{c}(L)\right), C\left(T_{c}(L)\right) / R$, and $\chi_{S}^{d}\left(T_{c}(L)\right)$ evolve as power laws with the size of the system and present good correspondence with the behavior expected for the three dimensional Ising model [27] (gray lines).

and a steep rise in the double monopole staggered density as a function of temperature (not shown), suggesting a phase transition. In this case the behavior of these quantities is typical of a second order transition for each value of $J_{n n} / D_{n n}$ studied, with no deltalike peaks or discontinuities.

In Fig. 7 we show the peak in the the molar specific heat for $J_{n n} / D_{n n}=-1.064$ and different sizes of the system. The inset shows that its value at the critical temperature, as well as the order parameter $\rho_{S}^{d}$ and its fluctuations $\chi_{S}^{d}$, evolves with size as a power law. As expected, we determined that the transition again belongs to the three-dimensional Ising universality class. Furthermore, since only one energy scale is present in this model [see Eq. (2)], the $T$ vs $\rho$ phase diagram shown in Fig. 8 does not depend on $T / D_{n n}$.

The dependence of the critical temperature on the relative strength of the exchange and dipolar interactions has already been explored for the DSIM [24,25]. In order to explicitly show the effect of dipolar interactions, we now present our version of the $T / D_{n n}$ vs $J_{n n} / D_{n n}$ phase diagram for both the DSIM and the NNSIM (Fig. 9). The first thing we note is that in the DSIM the staggered charge fluid extends to a region with small but ferromagnetic $J_{\text {eff }}$ (or, equivalently, $J_{n n} / D_{n n} \gtrsim-1$ ); this does not happen in the NNSIM. This can be easily understood within the monopole picture: In the same way as neutral atoms of $\mathrm{Na}$ and $\mathrm{Cl}$ ionize to form $\mathrm{NaCl}$, the energy spent in the creation of the monopoles can be compensated by the attraction between them. As a matter of fact, comparing the Madelung energy of a zinc-blende lattice of oppositely charged double monopoles and the energy needed to create these charges out of the vacuum [4] results in a zero-temperature limiting value of $J_{n n} / D_{n n} \approx-0.918$ [23], which is reasonably close, within the monopole picture approximation, to the value -0.905 found by us (Fig. 9) and Ref. [25]—both in spin systems. This quantitative agreement provides additional support for the monopole picture of spin ice.

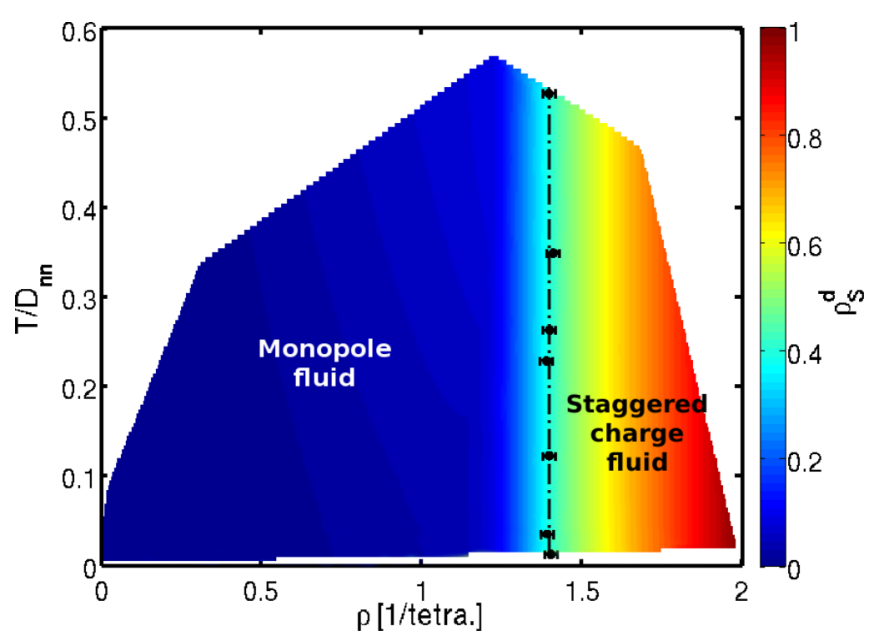

FIG. 8. (Color online) Temperature vs monopole density phase diagram with nearest-neighbors interactions. The second order phase transition (dot-dashed line) between the gas and the staggered charge fluid occurs at $\rho_{c} \approx 1.4$ at all temperatures, hence we call it a geometric phase transition. The diagram is overlaid on top of an interpolated contour plot of $\rho_{S}^{d}$ for $L=4$.

\section{ROLE OF THE SPIN DEGREES OF FREEDOM}

\section{A. Charge interactions vs correlations}

The strength of the monopole picture, that allows not only a qualitative but also a quantitative understanding of the phase diagram, seems at first sight to be weakened by a plain fact: No Coulomb attraction between oppositely charged monopoles is expected in the NNSIM (note that we

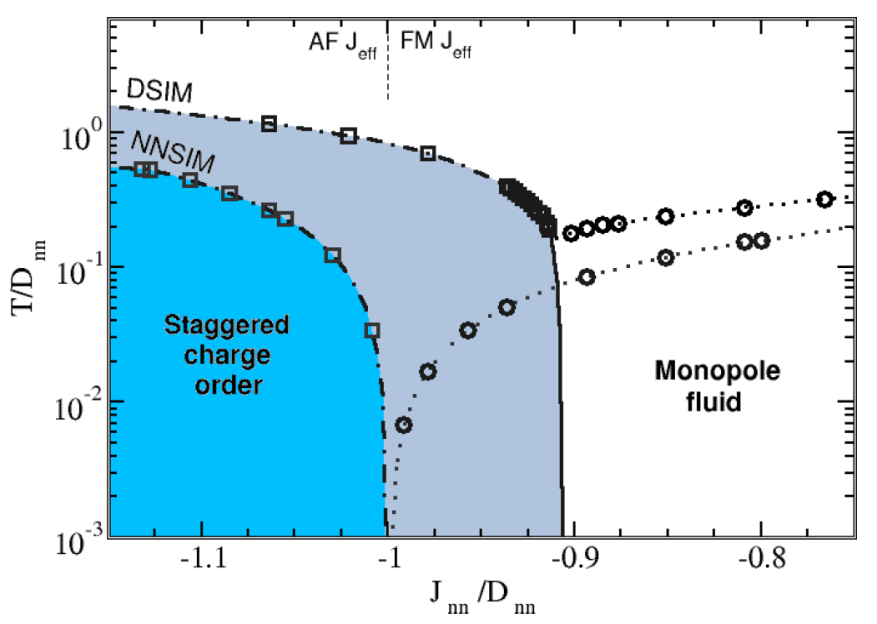

FIG. 9. (Color online) $T / D_{n n}$ vs $J_{n n} / D_{n n}$ phase diagram for both dipolar and nearest-neighbors spin ice models. Dotted lines represent crossovers, while solid and dot-dashed lines stand for first and second order phase transitions, respectively. The effect of the dipolar interactions is clearly seen: The system orders at the alternating charge configuration even for values of $J_{\text {eff }}>0$, because the Coulomb attraction between monopoles of opposite charge makes it energetically favorable to create and order these particles. Melko-Gingras-den Hertog first order transition [25] is not visible because we used a single spin-flip algorithm that mimics the real material and thus freezes at low temperatures. 


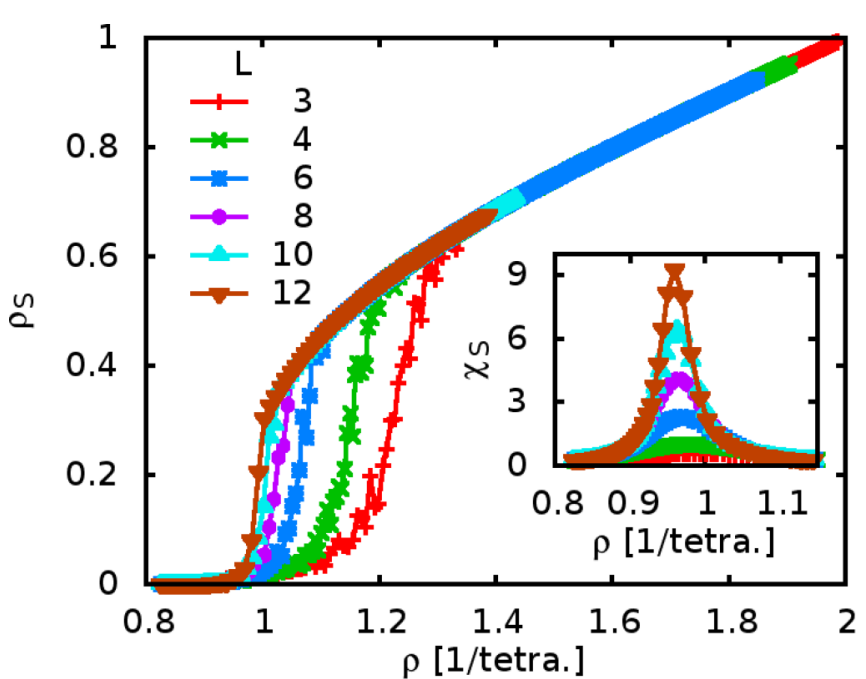

FIG. 10. (Color online) Staggered charge density (main figure) and its fluctuations (inset) vs particle density for a system of "plus" and "minus" noninteracting hard spheres within the Grand Canonical Ensemble. Finite size scaling of these quantities is consistent with the three dimensional Ising universality class [27] (not shown).

continue calling the excitations "monopoles" despite the fact that they have lost their main quality as charges). While it is simple to understand why the spins would order in the alternating "all-in-all-out" configuration when only a negative nearest-neighbors interaction $J_{\text {eff }}$ is considered (Fig. 9), it is much harder to rationalize, limiting ourselves to the monopole picture, why nonattracting monopoles would experience any sort of staggered ordering.

This simple puzzle can be solved by taking into account the correlations between monopoles, which transcend any Coulomb-like interactions. It is not energetic disfavor but construction constraints that prevent more than one double charge in a single diamond site. In the same way, the underlying spin configuration ("all-in" or "all-out") forbids two double monopoles with the same double charge to be placed in adjacent tetrahedra. A similar reason makes it more probable to find single monopoles with the opposite charge around any double monopole. Provided there are enough double charges in a diamond lattice, this correlation, quite equivalent to an infinite repulsion between double charges of the same sign at nearest neighbor sites, will induce staggered ordering on the system, irrespective of the system temperature.

In order to test this point quantitatively, we have simulated a system of nominal "plus" and "minus" hard spheres in the diamond lattice within the Grand Canonical Ensemble. In analogy to the NNSIM, we included no interactions between spheres, but a constraint was imposed forbidding two like spheres to be placed in neighboring sites. Figure 10 shows the staggered charge density $\rho_{S}$ for the spheres as a function of total average sphere density [30] $\rho$, for different system sizes; a cubic unit cell of side $L$ is again implied. The sudden increase in $\rho_{S}$ reflects the chargelike order being stabilized in the system as a consequence of sphere correlations. A previously known example of this kind of symmetry breaking in hard-sphere systems can be found in binary mixtures with a radius ratio of $R_{A} / R_{B} \approx 0.4$ and 0.76 , where $R_{A}\left(R_{B}\right)$ is the radius of the small (large) spheres. Driven by differential excluded volume effects, these systems present $\mathrm{NaCl}$ and $\mathrm{CsCl}$ structures, respectively $[31,32]$, in which every $A$ particle is surrounded only by $B$ particles, and vice versa, resembling the staggered ordering of our noninteracting double "monopoles."

Once more, finite size scaling of the order parameter and its fluctuations (Fig. 10, inset) allowed us to identify the three dimensional Ising universality class. The extrapolated critical density $\rho_{c}(L \rightarrow \infty)=0.964 \pm 0.004$ is near the critical value found for $\rho_{c}$ in Fig. 8 for the NNSIM. The differences between these two critical monopole concentrations can be explained realizing that our simple sphere model does not consider single monopoles, which add extra charge with smaller correlations. Considering then that even the NNSIM involves an effective contact interaction between monopoles, we can now understand the independence of $\rho_{c}$ with temperature observed in Fig. 8 as a result of a construction constraint.

This reasoning gains in depth when we contemplate its implications to the dipolar model studied before. While the low temperature region of the phase diagram in Fig. 6 is, as discussed, obviously dominated by the Coulomb attraction between monopoles, the ordered phase seen for $T / D_{n n} \gg 1$ at high monopole density can now be trivially explained in terms of the aforementioned charge correlations. Indeed, the vertical asymptote we noticed in Fig. 6 shows the equivalence between the DSIM and NNSIM in the limit of high $T / D_{n n}$.

As mentioned, correlations beyond charge interactions are much smaller for single charges. Indeed, using our conserved-monopole algorithm [22] for nearest neighbors spin interactions we have proved that a system of single monopoles remains charge disordered, even in the limit of $\rho=1$. This explains the observation of a high temperature limit for the stability of the fluid of single monopoles observed in Ref. [22].

\section{B. Discussion: Re-entrance near the tricritical point}

Figure 6 suggest the re-entrance of the charge-disordered phase near the dome. Indeed, we have observed that this behavior persists for bigger lattices, of which we have explored up to $L=5$. Though not in a conclusive way, this suggests that this behavior is not the mere consequence of the tricritical fluctuations. As in the previous discussion, we propose an explanation for the re-entrant behavior, which is not observed in the Blume-Emery-Griffiths model [26] nor in lattice models of real charges [29,33] nor in the CDSIM [22], which transcends the mere chargelike degrees of freedom.

A clue of the entropic origin of the re-entrance can be obtained by noticing that the charge-ordered phase extends its region of stability on increasing temperature, reaching lower densities. We have checked that along the transition line the reduction in $\rho$ is related to an increase in the number of empty ("2-in/2-out") tetrahedra, while keeping an approximately constant concentration of single-charge monopoles. This mechanism, which necessarily decreases the number of double defects, is very efficient in increasing the entropy of the system. This entropic contribution, which can be related to internal degrees of freedom of the charges, can outweigh the expected decrease in energy, reducing the free energy of the staggered phase. The balance is no longer possible for low enough densities, and the previously observed 
chargelike behavior $[22,29,33]$ is recovered, with $\rho_{c}$ increasing with temperature.

\section{Charge order with spin disorder: Best chance within an Ising pyrochlore material}

We have already mentioned the impossibility of stabilizing in a real Ising pyrochlore material a perfect single-monopole crystal at zero magnetic field. As stated in Ref. [23], such a crystal would imply the existence of charge order coexisting with a Coulomb phase. We believe that the staggered chargeordered phase near the re-entrant region is the closest we can get in this system to this situation. At $T / D_{n n} \approx 0.57$ and $\rho \approx 1.1$ (see Fig. 1 for a snapshot of a part of the system at these values) almost $50 \%$ of the diamond lattice sites are occupied by single defects, while only $30 \%$ are double monopoles. This leftmost transition point, in which the staggered charge order is impending, is quantitatively compatible with the phase diagram obtained for the CDSIM (since only single charges are allowed in this model, a double monopole in the DSIM must be equated to a single monopole in the CDSIM, then the total density $\rho=1.1$ in the first model corresponds to $\rho \approx 0.5+0.3=0.8$ in the latter). Indeed, Fig. 4 in Ref. [22] shows that for $80 \%$ occupation staggered order is established near $T / D=5 / 3 T / D_{n n} \approx 0.95$, plainly consistent with the value $T / D_{n n} \approx 0.57$ we find for the unrestricted DSIM. This coincidence implies that double monopoles for this level of dilution contribute to the staggered-charge order similarly to single monopoles. An important consequence of this fact is that a neutron scattering measurement of an Ising pyrochlore material with $J_{n n} / D_{n n} \approx-0.947$ at $T \approx 0.57 D_{n n}$ (so as to have $\rho \approx 1.1$ inside the ordered phase, but with a significant fraction of single-charged monopoles) should give a structure factor quite similar to that simulated in Fig. 4 of Ref. [23]. This pattern shows signs of spin fragmentation, combining Bragg peaks from an "all-in-all-out" structure with the pinch points which characterize the underlying Coulomb phase.

\section{CONCLUSION}

The path we have taken in this paper has been twofold. First, we have shown how the monopole picture arises naturally as a tool to reinterpret and understand the physics of dipolar Ising pyrochlore systems, encompassing both spin ice and antiferromagnetic materials. The magnetic phase diagram for classical Ising pyrochlores calculated by den Hertog and collaborators [24], separating the "all-in-all-out" antiferromagnet from the spin ice systems in terms of the effective exchange constant values, has been recast into temperature vs density, in analogy to the phase diagrams in molecular systems. Notably, in spite of its underlying spin nature and the presence of four types of different nonconserved magnetic charges (plus and minus, single and double), the dipolar model gives rise to a phase diagram which is quite comparable with those previously obtained for on-lattice systems of electric charges [29], and on spin ice models with a conserved number of single magnetic charges [22]. Thinking just on monopoles and their interactions made it simple to justify quantitatively the extent to which the antiferromagnetic phase gets into the region of ferromagnetic first neighbors effective exchange interaction, by evaluating the Madelung energy of a double monopole crystal. This plainly exemplifies how the power of the picture does not limit itself to the qualitative understanding of the phases present. Within the nearest-neighbors model, the stabilization of the antiferromagnetic phase was interpreted within the monopole picture in terms of the proliferation of monopoles with no Coulomb interactions but which implicitly force a nearest-neighbor exclusion condition between like types. This exclusion condition mimics an attraction/repulsion between like/different charges, maintaining the idea of monopole as a useful concept even within the NNSIM.

We also encountered certain aspects on the phase diagrams which cannot be explained in terms of simple charges. Like the discovery of internal degrees of freedom in particles previously thought of as indivisible building blocks, these findings are far from making the monopole picture less interesting. Among these peculiarities we found a re-entrance of the disordered fluid, and a staggered charge-ordered fluid phase which-for high enough densities - can be stable at temperatures arbitrarily much higher than the energy scale characterizing charge interactions. This second fact could be understood in terms of construction constraints- the exclusion condition we referred to in the previous paragraph-inherent to the spin nature of the excitations. The local constraint (as opposed to Coulomblike monopole attraction) is the predominant correlation mechanism between monopoles at high temperatures and high monopole density, and the only interaction for the NNSIM. We explained the re-entrance noticing that lowering the density of double charges induces a reduction in the energy but a boost in this energy's degeneracy. A final remark is the identification near the re-entrance of a good candidate for a state where charge order can coexist with a Coulomb phase [23] (an Ising pyrochlore material with $J_{n n} / D_{n n} \approx-0.947$, at $T / D_{n n} \approx 0.57$ ). The scattering pattern of this state should show signs of spin fragmentation, combining Bragg peaks from an "all-in-all-out" structure with the pinch points which characterize the underlying Coulomb phase [23].

\section{ACKNOWLEDGMENTS}

We thank T. S. Grigera for helpful discussions. This work was supported by Consejo Nacional de Investigaciones Científicas y Técnicas (CONICET) and Agencia Nacional de Promoción Científica y Tecnológica (ANPCyT), Argentina.
[1] P. Anderson, Concepts in Solids: Lectures on the Theory of Solid, World Scientific Lecture Notes in Physics (World Scientific, New Jersey, 1997).
[2] D. Khomskii, Basic Aspects of the Quantum Theory of Solids: Order and Elementary Excitations (Cambridge University Press, Cambridge, 2010). 
[3] F. Steglich, J. Aarts, C. D. Bredl, W. Lieke, D. Meschede, W. Franz, and H. Schäfer, Phys. Rev. Lett. 43, 1892 (1979).

[4] C. Castelnovo, R. Moessner, and S. L. Sondhi, Nature (London) 451, 42 (2008).

[5] D. J. P. Morris, D. A. Tennant, S. A. Grigera, B. Klemke, C. Castelnovo, R. Moessner, C. Czternasty, M. Meissner, K. C. Rule, J. U. Hoffmann, K. Kiefer, S. Gerischer, D. Slobinsky, and R. S. Perry, Science 326, 411 (2009).

[6] H. Kadowaki, N. Doi, Y. Aoki, Y. Tabata, T. J. Sato, J. W. Lynn, K. Matsuhira, and Z. Hiroi, J. Phys. Soc. Jpn. 78, 103706 (2009).

[7] T. Fennell, P. P. Deen, A. R. Wildes, K. Schmalzl, D. Prabhakaran, A. T. Boothroyd, R. J. Aldus, D. F. McMorrow, and S. T. Bramwell, Science 326, 415 (2009).

[8] I. Ryzhkin, J. Exp. Theor. Phys. 101, 481 (2005).

[9] L. D. C. Jaubert and P. C. W. Holdsworth, Nat. Phys. 5, 258 (2009).

[10] D. Slobinsky, C. Castelnovo, R. A. Borzi, A. S. Gibbs, A. P. Mackenzie, R. Moessner, and S. A. Grigera, Phys. Rev. Lett. 105, 267205 (2010).

[11] B. Klemke, M. Meissner, P. Strehlow, K. Kiefer, S. A. Grigera, and D. A. Tennant, J. Low Temp. Phys. 163, 345 (2011).

[12] O. A. Petrenko, M. R. Lees, and G. Balakrishnan, J. Phys.: Condens. Matter 23, 164218 (2011).

[13] L. D. C. Jaubert and P. C. W. Holdsworth, J. Phys.: Condens. Matter 23, 164222 (2011).

[14] C. Castelnovo, R. Moessner, and S. L. Sondhi, Phys. Rev. B 84, 144435 (2011).

[15] A. Sen and R. Moessner, arXiv:1405.0668.

[16] S. T. Bramwell and M. J. P. Gingras, Science 294, 1495 (2001).

[17] J. D. Bernal and R. H. Fowler, J. Chem. Phys. 1, 515 (1933).

[18] C. L. Henley, Annu. Rev. Condens. Matter Phys. 1, 179 (2010).

[19] K. Matsuhira, C. Sekine, C. Paulsen, M. Wakeshima, Y. Hinatsu, T. Kitazawa, Y. Kiuchi, Z. Hiroi, and S. Takagi, J. Phys.: Conf. Ser. 145, 012031 (2009).
[20] H. D. Zhou, S. T. Bramwell, J. G. Cheng, C. R. Wiebe, G. Li, L. Balicas, J. a. Bloxsom, H. J. Silverstein, J. S. Zhou, J. B. Goodenough, and J. S. Gardner, Nat. Commun. 2, 478 (2011).

[21] H. D. Zhou, J. G. Cheng, A. M. Hallas, C. R. Wiebe, G. Li, L. Balicas, J. S. Zhou, J. B. Goodenough, J. S. Gardner, and E. S. Choi, Phys. Rev. Lett. 108, 207206 (2012).

[22] R. A. Borzi, D. Slobinsky, and S. A. Grigera, Phys. Rev. Lett. 111, 147204 (2013).

[23] M. E. Brooks-Bartlett, S. T. Banks, L. D. C. Jaubert, A. HarmanClarke, and P. C. W. Holdsworth, Phys. Rev. X 4, 011007 (2014).

[24] B. C. den Hertog and M. J. P. Gingras, Phys. Rev. Lett. 84, 3430 (2000).

[25] R. G. Melko and M. J. P. Gingras, J. Phys.: Condens. Matter 16, R1277 (2004).

[26] M. Blume, V. J. Emery, and R. B. Griffiths, Phys. Rev. A 4, 1071 (1971).

[27] K. Huang, Statistical Mechanics (John Wiley \& Sons, New York, 1987).

[28] It should be stressed that the right part of this diagram corresponds to a single phase. The use of different names for two regions of it, which follows Ref. [22], does not imply that a different symmetry is broken but intends to emphasize their distinct dynamical properties and the fact that, while the double monopole crystal may coexist with a low density charge-disordered fluid, the staggered charge fluid (composed of both single and double monopoles) is homogeneous and occupies the whole space.

[29] R. Dickman and G. Stell, AIP Conf. Proc. 492, 225 (1999).

[30] In order to make a closer analogy between the hard spheres and the double charges, we have given each sphere a value of 2 in number, thus reaching a saturation value for the density of $\rho=2$.

[31] J. K. Kummerfeld, T. S. Hudson, and P. Harrowell, J. Phys. Chem. B 112, 10773 (2008).

[32] L. Filion and M. Dijkstra, Phys. Rev. E 79, 046714 (2009).

[33] V. Kobelev, A. B. Kolomeisky, and M. E. Fisher, J. Chem. Phys. 116, 7589 (2002). 